\title{
Integrating Solid Cutting Simulation And Error Verification For Surface Machining Application
}

\author{
Hsin-Yu Cheng, Jo-Peng Tsai \\ Far East University \\ Taiwan \\ hycheng@cc.kuas.edu.tw
}

\author{
Yung-Chou Kao \\ National Kaohsiung University of Applied Sciences \\ Taiwan \\ yckao@cc.kuas.edu.tw
}

\begin{abstract}
In this paper, a method integrating NC decoding and solid milling simulation to solve this problem is proposed. A modified Z-Map computational algorithm is adopted and it directly uses straight line and arc interpolation algorithms to calculate mesh position and $Z$ coordinates of tool scanning surface according to tool path and initial tool scanning boundary in reducing the long computation time. The proposed method in this paper includes NC decoding, solid cutting simulation, virtual measuring, and the residual material calculation in previous process. This method owns the advantage of no need to solve non-linear equation any more. Subsequently, through comparing the product surface with the data decoded from the NC program, the magnitude and the position of the machining geometry error could be calculated to serve as important reference for the machining process of complex geometry such as a 3D surface. An example was demonstrated to the feasibility of the proposed method.
\end{abstract}

Keywords-virtual measuring, NC simulation, Z-Map, intelligent machining

\section{INTRODUCTION}

Solid cutting simulation owns the capability to predict the geometry of machined work-piece. However, only in the condition not considering the errors due to machine, motion control and tool, the machined geometry can be correctly calculated by simulation software. In this paper, through comparing the geometry of the product with the machined surface, the machining error is acquired in the condition not considering the machine and tool deformation. In addition, since the finishing amount is related to the residual material after rough machining, particularly in the $3 \mathrm{D}$ sculptured surface machining, the irregular residue due to rough machining would result in uneven machining volume, consequently cause tool chatter which would worsen the finishing surface quality. In order to avoid such instability, the method proposed in this paper adopts solid cutting simulation and virtual measuring to calculate the residue in each machining step, so as to improve the unstable machining.

In order to know the position of machining error as well as the corresponding $\mathrm{NC}$ program, it is necessary to decode the $\mathrm{NC}$ program and detect where the NC program of the machining error is. Therefore, the machining process could be modified so as to improve the machining precision. These applications could provide the NC machining process with intelligent functions, that is, these featured functions could help engineer to detect the expected result before the actual machining and to show the location of the error and suggest the position to be improved. In this paper, we adopted NCcode decoding, solid cutting simulation, residue calculation, and virtual measurement of machined work-piece to implement these functions.

\section{LITERATURE REVIEW}

There are a lot of commercial software for NC machining simulation and verification available in the market, such as VericutTM, and a variety of CAD/CAM software such as CATIATM and SurfCAMTM. The methodology underlying these software are either simulated with image-based, objectbased or solid-based methods. The software of object-based three-axis simulation often adopts Z-Map method, which is most commonly used in machining simulation, especially in mold machining; this might be that $\mathrm{Z}$ direction is visual surface in Z-Map method, which is related to the direction of mold drafting.

There are many studies on Z-Map method. Ding [1] simulated the machining of flat-end and ball-end mill. A cutting profile sweeping contour of a general tool has been derived by Chung et al. [2], and the derived two-variable surface has also been simplified into a 4-degree single variable parametric curve to solve the projection profile resulting in the Z-Map value. This 4-degree parametric curve has been further converted by Maeng [3] into a quadratic single-variable nonlinear curve to simplify the calculation.

In order to reduce the calculation time, Lee et al. [4] proposed a subdivision method for the meshes covering the intersectional boundary profile between the moving cutter and the work-piece, while the meshes of other parts were remained the original division. Such an approach could sustain simulation precision and shorten computing time. Moreover, Yun [5] also proposed a method to move the meshes crossing boundaries so as to make the locations of border meshes and real borders in line. A better precision could then be obtained without increasing the number of meshes. Du et al. [6] used the envelope theory and swept volume generation method for a 5-axis solid milling simulation as well.

To enhance display quality and speed, Liu et al. [7] proposed to record multiple segmented quadrilaterals in quad-tree data structure of the work piece surface. After tool processing, the surface enveloped by the tool was re- 
segmented or removed of nodes to form the new polygons in accordance with allowable errors. Hence, the number of quadrilaterals can be reduced without affecting the display quality. Mao [8] employed the similar concepts to reallocate the grids of the triangle mesh according to local surface flatness to reduce number of grids and obtain the same display effects.

For the machining simulation integrated with cutting force estimation, Yun et al $[5,9]$ predicted cutting force for the initial transient toolpath and the stable middle toolpath of flat-end milling tool based on Z-Map method. Fussel et al [10] also estimated cutting force on five-axis sculptured surface milling operation.

\section{RESEARCH METHOD}

In general, the theory of solid milling has been widely applied to various commercial CAM software successfully, but it seems that there is still no related research in combining it with virtual measurement and NC decoding. Therefore, this paper aimed at integrating $\mathrm{NC}$ decoding function into solid milling simulation, and a virtual measuring function has also been integrated in order to compare the designed product surface and the machined surface for the reason of showing the machined error. Furthermore, Direct-3D graphics library was adopted to visualize the machined geometry and geometrical errors in different colors; this will enable a user to easily view the location of the error area for subsequent necessary complementary cutting.

\section{A. The application of interpolation algorithm in Z-Map}

Different tool swept surfaces will be generated according to different tools and $\mathrm{NC}$ paths (e.g., straight-line cutting or arc cutting), so it is necessary to derive different projection equations for different tool shapes. Figure 1 shows the envelope surface produced by a ball-end mill and Figure 2 is the calculated projection depth, through which the machined surface would be generated after calculating each NC blocks in the tool path. Figure 3 shows the tool path of a mold in rough machining and Figure 4 is the simulation result of the tool path with Z-Map method.

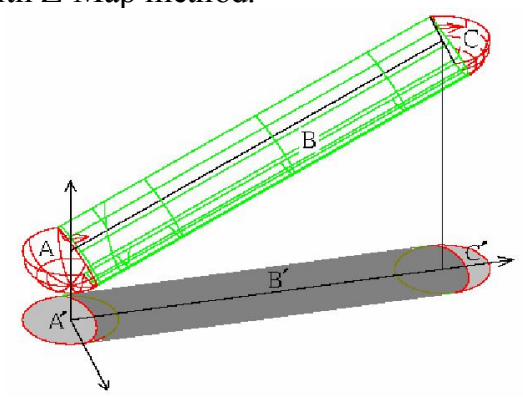

Figure 1. The envelope surface of ball end mill during straight-line cutting

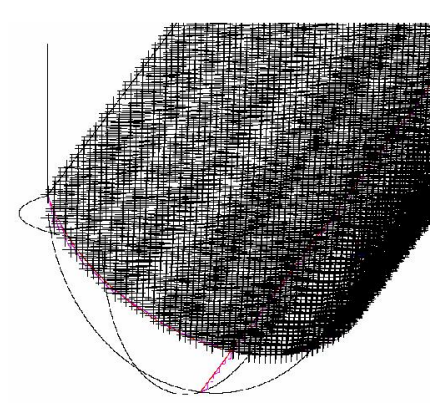

Figure 2. The projection depth of tool swept surface

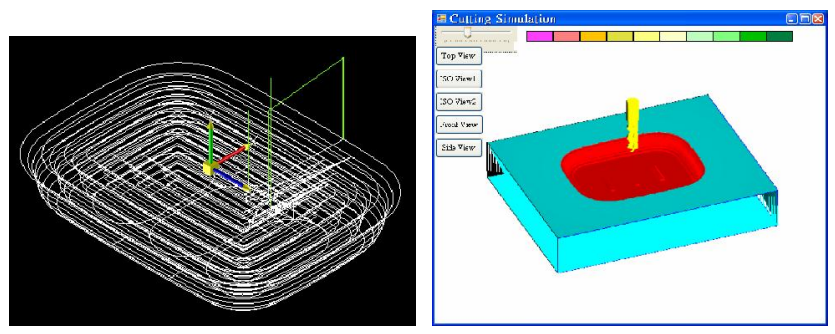

Figure 3. The tool path of a mold rough machining (left) and simulation result of the tool path with Z-Map(right)

( $\Phi 16 \mathrm{~mm}, \mathrm{r} 2$, bull-nose end mill)

As the use of Z-Map for cutting simulation requires calculating the $\mathrm{Z}$ coordinates of all grid points in the range of tool scanning when each executing each line of the $\mathrm{NC}$ program, the computation sum is very large. As a result, if the coordinates of each grid point have to be calculated by the inferred mathematical program, it is time-consuming. Therefore, this paper proposes the interpolation algorithm to simplify the calculation based on the following principles: (1) first calculate the areas generated by the scanning of the tool in fixed positions without movement in case of each line of the NC program (it is the starting and ending positions of the tool, see $\mathrm{A}$ and $\mathrm{C}$ areas in Figure 1) and the grid point location of the contact border of the tool movement area (Area B) as well as the Z coordinates; (2) then, conduct interpolation calculation of the tool path according to grid size; (3) according to the movement mode of the interpolation movement, calculate the $\mathrm{Z}$ coordinates of each movement location in proportion rather than calculation, according to the inferred equations. Hence, there is no need to calculate tool scanning border or search for the tool scanning border range. The proposed method can save huge amount of calculation time.

\section{B. Automatic virtual measurement}

In order to check the optimization of machining result and NC cutting process, it is necessary to understand the cutting parameter setting of each procedure, which is the most difficult part for complex 3D surface machining such as in mold cutting. General speaking, it is determined by engineers possessing considerable experience. One of the keys in determining the cutting parameters is to understand the removal amount of material and the distribution of the related residual material. Therefore, this paper developed the method integrating solid cutting simulation with the automatic virtual measurement, with which the engineers 
could know whether tools and the machining position were properly selected in each procedure or not, so that the removed material in each procedure is identical, so as to lessen the tool chatter or tool excessive deformation. Especially in finish machining, it is necessary that the load of tool is in a reasonable range, rather than modify it when the problem occurs after actual machining.

The calculation procedures of automatic virtual measurement are: (1) firstly decode the $\mathrm{NC}$ program to get its tool path through which to calculating the solid milling simulation with Z-Map Theory, and (2) use Direct3D to show the dynamic machining result. The virtual error measurement requires importing the compared surface for the correct calculation of the measuring points. Then it is compared with the calculated result of the cutting simulation to obtain the virtual machining errors, which are identified by a variety of colors. The process is shown in Figure 4. By way of this method, users can see the magnitude and location of the error, so that $\mathrm{NC}$ generation engineer can understand whether the cutting conditions and the cutting location were properly selected for each procedure.

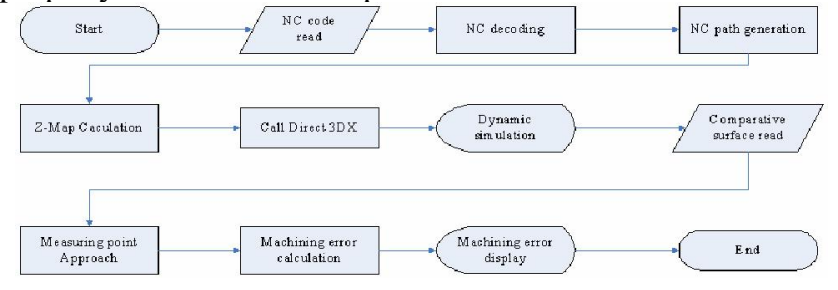

Figure 4. The flowchart of virtual machining error measurement

\section{IMPLEMETATION}

This paper adopted a mold machining example to verify the results of this study, and discussed its applications towards intelligent machining. The characteristic of this example is that there is a small fillet groove (R2.5) located on the vertical surface. As the groove is in the inclined plane, it can not be machined with a large-diameter bull-nose mill. Moreover, since the bottom of the groove is $18 \mathrm{~mm}$ lower than the flange, it is prone to generate chatter if a $5 \Phi$ ballend mill is used, which suspend $20 \mathrm{~mm}$ tool-length outward the chunk. As a result, the volume of removed material at each process should be carefully set in order to avoid errors due to tool deformation; otherwise, a coarse surface would be generated due to chatter. In this paper, the machining result of the virtual measurement will be used for the basis of predicting the machining amount of each process.

Experimental machining process was divided into several steps: (1) rough milling: a $25 \Phi \mathrm{mm}$ flat-end mill was used for $\mathrm{Z}$ roughing, and then (2) did planar milling. The residue was set as $0.5 \mathrm{~mm}$ for the two previous steps. The tool path after decoding the NC program and the corresponding solid milling simulation is shown in Figure 5. In this figure, it reveals a more protruding part would have more material residues although the amount is unknown. Therefore, it is difficult to select appropriate tool and set the finishing amount for the next step. For this reason, this research developed automatic virtual measuring enable users to clearly see the magnitude and distribution of errors, which are used as a reference for the next step. Automatic detection after virtual machining requires importing the product surface in advance, onto which the measuring points are projected. Figure 6 shows the data projected onto the product surface and become line segments for comparison. In this experiment, the pitch of measuring points was $0.5 \mathrm{~mm}$, thus 63,052 milling measuring points were calculated.

TABLE I. PART OF THE MEASURING POINTS (1)

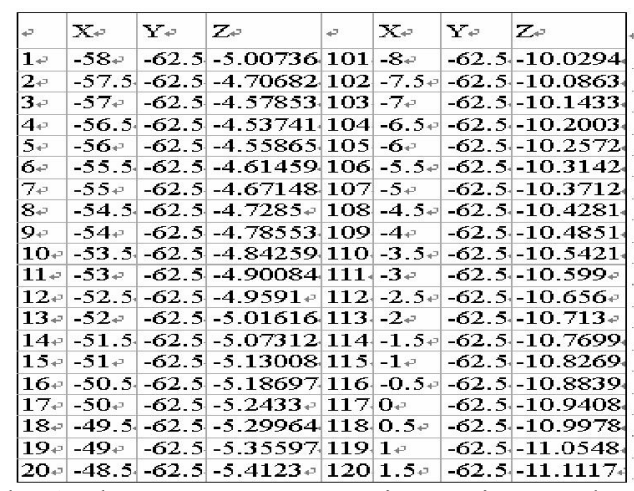

Table 1 shows some measuring points and Figure 7 shows the error distribution after rough milling. The residue was set as $0.5 \mathrm{~mm}$, therefore, most of the errors fell among $0.1 \sim 0.5 \mathrm{~mm}$. However, the errors were not totally identical with $\mathrm{CAD} / \mathrm{CAM}$ settings, as there were some locations at which tools could not arrive. A few errors were even above $1.5 \mathrm{~mm}$, especially for the locations close to the cliffs and grooves, while other errors occurred at the locations of round corners. Error values are shown in Table 2.

TABLE II. PART OF THE ERROR VALUES AFTER ROUGH MILLING IN PROCESS (1)

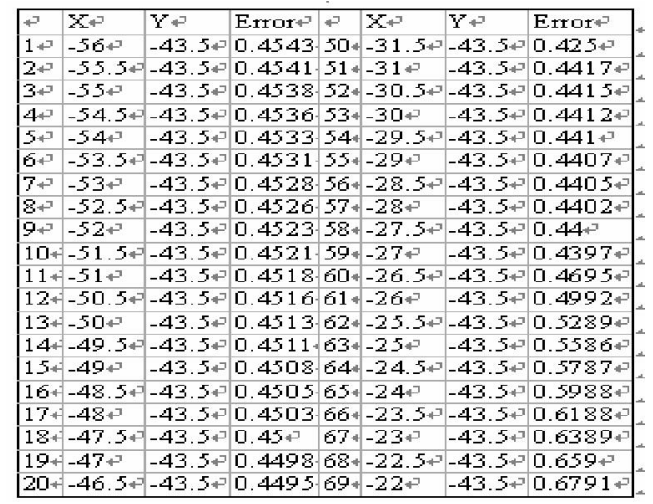

The next experimental process, step (2), used a $\Phi 20 \mathrm{~mm}$ ball-end mill to obtaining smaller residue. The machining result is shown in Figure 8, and the corresponding error distribution map of virtual measurement is shown in Figure 10 , from which it reveals that although the majority of machining accuracy fall below $0.05 \mathrm{~mm}$ as required, however, the error distribution is not improved considerably and the machining error distribution is almost the same as the step (1). The high residue is still located near the grooves. Figure 9 shows the result of virtual measurement after step (2) (at the section $\mathrm{Y}=0$ where is the location of work-piece center, 
to measure with $0.5 \mathrm{~mm}$ pitch along $\mathrm{X}$ axis). The maximum error occurs at $X=-37.0 \mathrm{~mm}$ where the error value is $2.17 \mathrm{~mm}$ which had to be carved with a $\Phi 5 \mathrm{~mm}$ ball-end mill, however, the problem mentioned in previous paragraph may occur because the tool needs to suspend $18 \mathrm{~mm}$ outward.
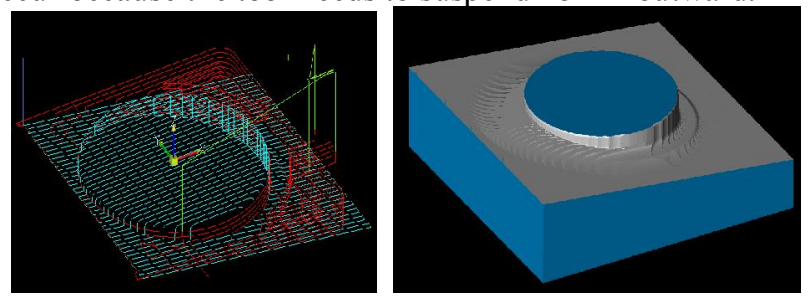

Figure 5. The Machining process of rough milling with tool path after decoding NC program(left), machining result of rough milling (right)

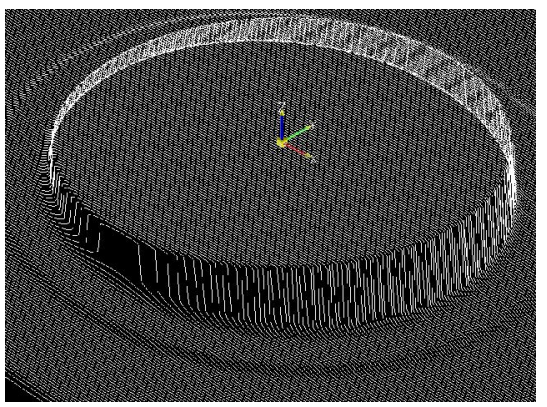

Figure 6. transform measuring points to line

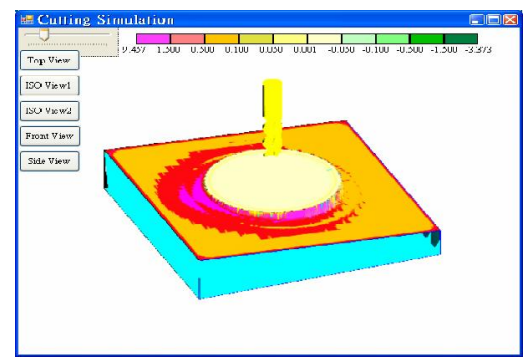

Figure 7. The error distribution map after rough milling in step

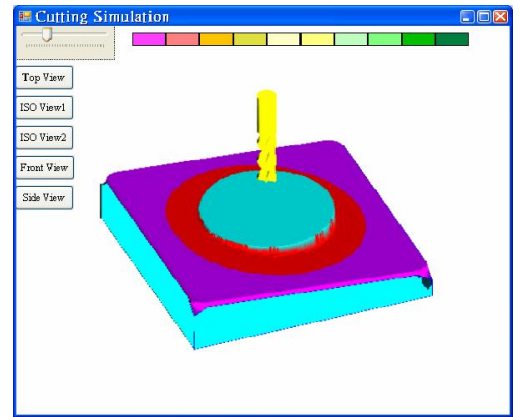

Figure 8. The result of machining simulation of step (2)
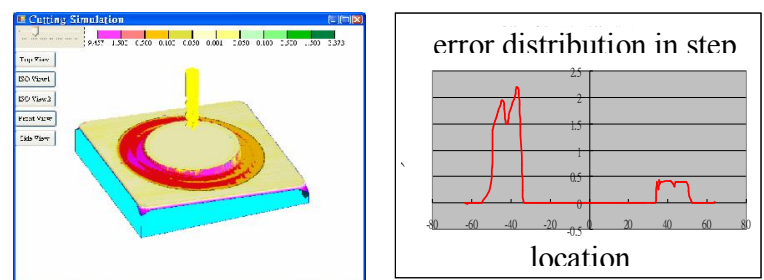

Figure 9. The error distribution map after rough milling(left) and the error values of virtual measurement after machining (right) in step (2)

\section{CONCLUSIONS}

The purpose of this paper was to demonstrate the innovative idea through a practical example through the synergy of solid milling simulation and virtual measurement in intelligent machining process. Generally, most of commercial $\mathrm{CAD} / \mathrm{CAM}$ software provides the function of solid milling simulation, but there is rarely the virtual measuring function with which to compare the product surface and the machined surface. Therefore, this paper not only proposed a method to integrate solid milling simulation, $\mathrm{NC}$ decoding and virtual measurement, but also applied them to the research of practical machining process. It provides an intelligent numerical machining process, rather than blindly complying with previous experience or the trial-and- error to improve machining process. The method proposed in this paper provides a feasible reference to decide the reliability and suitability for each machining step.

\section{REFERENCES}

[1] C. Y. Ding, "NC machining simulation under high-speed milling," master's thesis, Department of Mechanical Engineering, National Chung Cheng University, Chiayi, 2001.

[2] Y. C. Chung, J. W. Park, H. Shin, abd B. K. Choi, "Modeling the surface swept by a generalized cutter for NC verification," Computer Aided Design, vol. 30, no. 8, pp. 587-594, 1998.

[3] S. R. Maeng, N. Baek, S. Y. Shin, and B. K. Choi, "A Z-map update method for linearly moving tools," Computer Aided Design, vol. 35 , pp. 995-1009, 2003.

[4] S. K. Lee and S. L. Ko, "Development of system foe machining process using enhanced Z-map model," Journal of Material Processing Technology, vol. 130-131, pp. 608-617, 2002.

[5] W. S. Yun, J. H. Ko, H. U. Lee, and K. F. Ehmann, "Development of a virtual machining system, Part 3:cutting process simulation in transient cuts," International Journal of Machine Tools \& Manufacture, vol. 42, pp. 1617-1626, 2002.

[6] S. Du, T. Surmann, O. Webber, and K. Weinert, "Formulating swept profiles for five-axis tool motions," International Journal of Machine Tools \& Manufacture, vol. 45, pp. 849-861, 2005

[7] S.Q. Liu, S.K. Ong, Y.P. Chen, A.Y.C. Nee, "Real-time, dynamic level-of-detail management for three-axis NC milling simulation", Computer-Aided Design, Vol. 38 , pp. 378-391, 2006.

[8] Jian Mao, Shiqing Liu, Zhu Gao, "Three-axis NC milling simulation based on adaptive triangular mesh", Computers \& Industrial Engineering, Vol. 60, pp. 1-6, 2011.

[9] Won-Soo Yun, Jeong Hoon Ko, Han UI Lee, Kornel F. Enmann, "Development of a virtual machining system, Part 1:approximation of the size effect for cutting force prediction, International Journal of Machine Tools \& Manufacture, vol. 42, pp. 1595-1605, 2002.

[10] B.K. Fussell, R.B. Jerard, J.G. Hemmett, "Modeling of cutting geometry and force for 5-axis sculptured surface machining", Computer Aided Design, Vol. 35, pp. 333-346, 2003. 
The 2nd International Conference on Computer Application and System Modeling (2012)

Published by Atlantis Press, Paris, France.

(c) the authors 